\title{
The prevention and management of rabies
}

\author{
Natasha S Crowcroft chief, infectious diseases ${ }^{1}$, Nisha Thampi medical director ${ }^{2}$ \\ ${ }^{1}$ Public Health Ontario, 480 University Avenue, Suite 300, Toronto, ON, M5G 1V2, Canada; ${ }^{2}$ Infection Prevention \& Control, Division of Infectious \\ Diseases, Children's Hospital of Eastern Ontario, Canada
}

Rabies is a lyssavirus infection resulting in acute encephalitis or meningoencephalitis that is virtually always fatal. The disease can be caused by several different rabies and rabies-like viruses (box 1). Rabies is a neglected tropical disease that predominantly affects the most vulnerable humans-children living in the most disadvantaged areas of the poorest countries. ${ }^{1}$ Many countries have successfully reduced the impact of the disease by tackling the gap between public and animal health through a concerted "one health" approach. ${ }^{2}$

Clinicians worldwide need to be aware of rabies and vigilant about the possible exposure of patients to infection because timely prevention is life saving. The purpose of the review is to give an overview of rabies prevention and the management of patients who may have been exposed to infection or are suspected of having rabies.

\section{What is the global burden of rabies?}

Countries predominantly affected by rabies often have poor diagnostic and reporting capacities, leading to a lack of accurate data and considerable uncertainty around estimates of global burden. ${ }^{3}$ Efforts to improve data quality have been hampered by duplicative reporting systems requirements to different agencies representing animal or human health. In 2011 the World Health Organization reporting database Rabnet closed, as limited data and under-reporting contributed to a lack of priority for this disease. ${ }^{3}$

In the absence of high quality reporting, estimates of global burden in 2010 ranged from 26400 to 61000 deaths, depending on the method applied. Considerable geographical variation exists worldwide, with $95 \%$ of rabies cases in humans occurring in Africa and Asia; $84 \%$ of these in rural areas. ${ }^{2}$ Dogs are the source of infection in more than $99 \%$ of cases in humans.

The global economic burden of rabies has been estimated at \$6bn ( $£ 3.8 \mathrm{bn}$; $€ 5 \mathrm{bn}$ ), comprising the cost of both disease in humans, domestic animals, and wildlife and prevention through control measures in animals and post-exposure prophylaxis in humans. ${ }^{2}$ From a health policy maker perspective, control of canine rabies is highly cost effective and even cost saving. ${ }^{4}$ Post-exposure prophylaxis is also cost effective ${ }^{5}$ but expensive for individuals and does not contribute to public health measures to interrupt transmission. While post-exposure prophylaxis is usually publicly funded in developed settings, it may be unaffordable for individuals in the highest risk areas, and help may be sought from traditional healers. ${ }^{6}$ In India, the country with the highest number of deaths from rabies, only one in six patients receives appropriate post-exposure prophylaxis. ${ }^{2}$ In areas where canine rabies is eliminated, such as North America, the costs of post-exposure prophylaxis have not diminished because rabies continues to circulate in wildlife. ${ }^{7}$ For countries with good control programs, a challenge is to continue to be vigilant and to bear the costs of post-exposure prophylaxis given recurrent importations from neighboring countries with poor control, as seen recently in Greece. ${ }^{8}$

\section{What are the trends in global rabies control and elimination?}

Although there is uncertainty about, and under-reporting of, rabies, estimates are that the global burden of the disease decreased from 3234 to 1462 disability adjusted life years between 1990 and 2010. ${ }^{9}$

Vaccination of dogs is the key to primary prevention in humans. This is feasible even in the poorest parts of the world. ${ }^{10}$ Success relies on concerted multidisciplinary partnerships involving governments, animal and human health authorities, and a national reference laboratory to support diagnosis and surveillance.

Since rabies can be eliminated through vaccination of dogs, why do so many countries continue to have challenges in disease control? One reason is lack of communication between those responsible for human and animal health, including physicians and veterinarians. ${ }^{11}$

Terrestrial rabies has the potential to be eliminated, but this is unlikely to happen to bat rabies, which accounts for a small proportion of cases in humans each year. Bats are an essential part of the ecosystem and protected by law in many countries. Rabies vaccination is effective in bats, but there is no means of delivering it. 


\section{The bottom line}

Rabies remains a fatal disease in the majority of patients once symptoms develop

Treatment is supportive; protocols for curative therapy currently remain experimental

Given the extremely high mortality, prevention is of the utmost importance

Post-exposure prophylaxis through vaccine and immunoglobulin given soon after exposure to rabies virus is highly effective in preventing rabies

Rabies elimination requires concerted action by animal and human health authorities, focused on control of rabies in dogs and wild animals and timely prophylaxis for exposed people

\section{Box 1 Rabies and rabies-like viruses}

- Rabies virus - various strains found in terrestrial mammals worldwide (except for Australia, Antarctica, some islands), as well as bats in the Americas

- Australian bat lyssavirus_-bats in Australia and perhaps several nearby islands

- European bat lyssavirus types 1 and 2, Bokeloh bat lyssavirus, West Caucasian bat virus-bats in various parts of Europe

- Khujand virus, Aravan virus, Irkut virus-bats in Asia

- Duvenhage virus, Lagos bat virus, Shimoni bat virus, Mokola virus, Ikoma lyssavirus—bats or unknown host, Africa

\section{Sources and selection criteria}

In a non-systematic review of literature, we built on an existing collection of literature on rabies that had been accumulated since 2000 . We searched PubMed using MeSH term "rabies", limited to studies in English in humans conducted in the past five years. From that search we reviewed 846 abstracts for relevance and reviewed relevant papers in full, as well as reviewing bibliographies to identify further papers and searching key sources of grey literature, including the World Health Organization and national health authorities. The final list of references was also influenced by the scope and format of the article.

\section{Who is at risk?}

Those living in rabies endemic countries, without control measures in dogs and wildlife and access to post-exposure prophylaxis, are at greatest risk. Half of the human population worldwide lives in countries endemic for canine rabies (fig $1 \Downarrow$ ). ${ }^{2}$ Children are especially at risk because they are more likely to approach animals without caution, including apparently tame wild animals, and therefore to be attacked and bitten, especially on the arms and face. In rabies-free countries, all cases of terrestrial rabies are linked with importations (rabies-like viruses in bats do not affect a country's rabies-free status). For example, since 1946, 25 cases of human terrestrial rabies have been reported in the United Kingdom, associated with exposures to rabid dogs in countries such as the Philippines, Nigeria, India, and South Africa. ${ }^{12}$ Travelers and residents in rabies endemic countries should avoid contact with free-roaming animals, especially dogs.

Vampire bats have been associated with several rabies outbreaks in South America. Bats carry rabies-like viruses (rabies related lyssaviruses) even in terrestrial rabies-free countries in Europe and Australia. ${ }^{213}$ In 2002, a patient developed rabies after exposure to a bat, the only case acquired that way in the United Kingdom in over a century. ${ }^{14}$ In Canada, six of eight cases identified nationally since 1970 have been attributed to infections with rabies strains associated with bats. ${ }^{15}$ Although bats may be more important as reservoirs of rabies-like viruses than sources of infection in humans, people need to be aware of the risk of acquiring rabies from bats and seek immediate medical attention should they or their family members have contact.

\section{How is rabies transmitted?}

Lyssaviruses cannot cross intact skin. Rabies gets into the body through wounds or direct exposure of mucous membranes, usually as a result of bites from infected animals, or through transplantation of tissues or organs from someone who died from rabies (fig $2 \Downarrow$ ). Humans are an end host; anecdotal cases of transmission from human to human have not been confirmed outside of transplantation, ${ }^{16}$ including transmission from patients to healthcare workers.

The incubation period is variable, with a mean incubation time in one study of 273.6 days (median 80 days, range 12 days to 10 years). ${ }^{17}$ The longer incubation periods emphasize that post-exposure prophylaxis is always indicated even if exposure occurred months or years earlier, provided neurological symptoms have not developed.

\section{Can it be prevented?}

Rabies vaccination has been available for more than 125 years. ${ }^{18}$ Several modern cell culture and embryonated egg-based rabies vaccines (CCEEVs) containing inactivated rabies virus are available. Older nerve tissue vaccines should no longer be used as they may induce severe adverse reactions and are less effective than CCEEVs. Those who begin post-exposure prophylaxis with one of these older vaccines should restart the series with CCEEVs. ${ }^{2}$

\section{Pre-exposure vaccination}

Pre-exposure vaccination is strongly recommended for anyone who is at "continuous frequent or increased risk for exposure to the rabies virus." It is recommended that laboratory staff, veterinarians, and anyone who works with animals and wildlife receive pre-exposure prophylaxis to reduce their occupational risk of infection. ${ }^{19}{ }^{20}$ It should also be an important prevention strategy in other high risk groups, including infants and children in areas with a high incidence of canine rabies, ${ }^{21}$ and where access to immediate care or rabies immunoglobulin is limited. WHO recognizes two pre-exposure vaccination schedules; one administered intramuscularly and the other intradermally (box 2). Periodic boosters for immunized people are not usually recommended, apart from some workers at continual risk who should undergo serological monitoring and receive a booster if 
and when required. In the event of an exposure to rabies, post-exposure prophylaxis is still required for those who have received pre-exposure vaccination.

\section{Routes for administering vaccines}

In the WHO recommended schedules, the vaccines for both pre-exposure and post-exposure immunization can be administered by two routes: intramuscular and intradermal. The intramuscular route is universally recommended and most commonly used where resources are not a problem. The intradermal route has the advantages of being dose sparing, resulting in equivalent protection at up to $60-80 \%$ of the cost of the intramuscular route, and requiring a single visit for pre-exposure prophylaxis. The reduced costs increase the likelihood that patients will complete post-exposure prophylaxis. Intradermal immunization against rabies is used mainly in countries where WHO recommended vaccines have regulatory approval for this route of use. Disadvantages include the additional training required to ensure that the vaccine is administered correctly, and safety concerns about multi-use vials; the intradermal route is not recommended for pre-exposure prophylaxis in immunocompromised patients or those taking chloroquine for malaria treatment or prophylaxis. ${ }^{22}$

Greater awareness of the risks of undiagnosed encephalitis is needed in the context of organ transplant related rabies. Those who die of encephalitis of unknown cause ideally should be excluded from being donors. ${ }^{23}$ Antemortem diagnostic tests for rabies are not sufficiently reliable for screening to exclude rabies, nor is relying on a negative report of potential exposure. $^{24} 25$

\section{What measures should be taken after a possible rabies exposure (such as dog bite)?}

Post-exposure prophylaxis should be initiated immediately after exposure is suspected, especially if an unprovoked animal bites. Several different WHO approved schedules have been adopted for local use (table $1 \Downarrow$ and box 2); all include wound cleaning, followed by active and passive immunization.

Local recommendations should be followed since these are relevant to the approved vaccines and rabies immunoglobulin available in the vicinity. Expert advice is available in many countries through public health departments and national immunization guides (box 3). Post-exposure prophylaxis is highly effective in preventing the virus from reaching the nervous system. Few failures have been noted and most deviated from WHO recommended protocols. ${ }^{26}{ }^{27}$ Failures among those with complete pre-exposure immunization are even rarer if post-exposure immunization is given as per WHO protocols, with survival following documented exposure 20 years after the completion of pre-exposure immunisation. ${ }^{28}$ Post-exposure prophylaxis is considered futile when administered after the onset of clinical symptoms.

\section{Step 1: wound care}

Immediate wound cleaning greatly decreases the risk of developing rabies. Recommended first aid for bite wounds and scratches includes thorough flushing with soap and water, detergent, povidone iodine, or other virucidal substances. Care should be taken to avoid contamination or enlargement of the wound. A bleeding wound is a source of high risk infection. Rabies immunoglobulin must be infiltrated into the wound. Deferred surgical closure of the wound has been recommended, given case reports of post-exposure prophylaxis failures associated with primary repair. ${ }^{27}$

\section{Step 2: vaccination}

The decision on whether to initiate vaccination is sometimes difficult to make and may require expert advice (fig 2). A risk assessment should be guided by:

Location (country) of the potential rabies exposure - this helps to determine the likelihood of the animal being rabid. Post-exposure immunization may not be warranted if exposure occurred in a rabies-free country or region

Severity of exposure (table $2 \Downarrow$ )

Clinical features of the animal

Vaccination status of the animal and its availability for observation or testing (usually only applies to dogs, cats, and ferrets)

Species of animal (if known).

The decision about whether to initiate vaccination should be made quickly so that post-exposure vaccination can be started immediately and continued while the animal is being observed or pending the results of laboratory tests. Superficial scratches and bites, particularly by bats, should be taken seriously, as the bat rabies virus has been shown to replicate in epithelial cells. ${ }^{29}$ Even in the absence of a history of animal bites, the discovery of a bat in a room with a young person who cannot reliably report a bite, should raise concerns, especially when the person was sleeping. Assessment is required to rule out possible contact. Nevertheless, the likelihood of a case of human rabies after bat exposure without a bite or other close contact (for example, a bat flying into a room, observed by one or more adults, and safely removed or leaves without any direct contact) is extremely low.

If the animal in question is a dog, cat, or ferret and can be observed for 10 days, post-exposure vaccination may be started and discontinued if the animal remains well at the end of the observation period. While most countries use a five dose schedule, several have now adopted a WHO recommended four dose schedule, with vaccine administered intramuscularly at $0.1 \mathrm{~mL}$ on days $0,3,7$, and 14 (table 1 ). With this reduced schedule, rabies immunoglobulin is recommended for category II as well as category III exposures. If someone is immunocompromised, a fifth dose at day 28 is still recommended. ${ }^{30}$ Minor reactions at local injection site are common and more likely to occur after an intradermal vaccine, whereas serious adverse events, including Guillain-Barré syndrome and allergic reactions are rare. ${ }^{31}$

Specialized advice is needed for immunocompromised patients. They may need additional monitoring to assess whether an adequate immune response has been mounted after vaccination and whether additional boosters are indicated.

Vaccinated people only require two boosters, given intramuscularly on days 0 and 3, or intradermally in four doses at a single visit (table 1); no rabies immunoglobulin is required. ${ }^{2}$

\section{Step 3: rabies immunoglobulin}

Several different rabies immunoglobulin products are available but access to them is limited by global shortages and high cost. ${ }^{32}{ }^{33}$ Rabies immunoglobulin provides passive antibodies at the site of exposure. It is given once, as soon as possible, and within seven days after the first vaccine dose, before patients develop an active immune response. ${ }^{2}$ The recommended total 

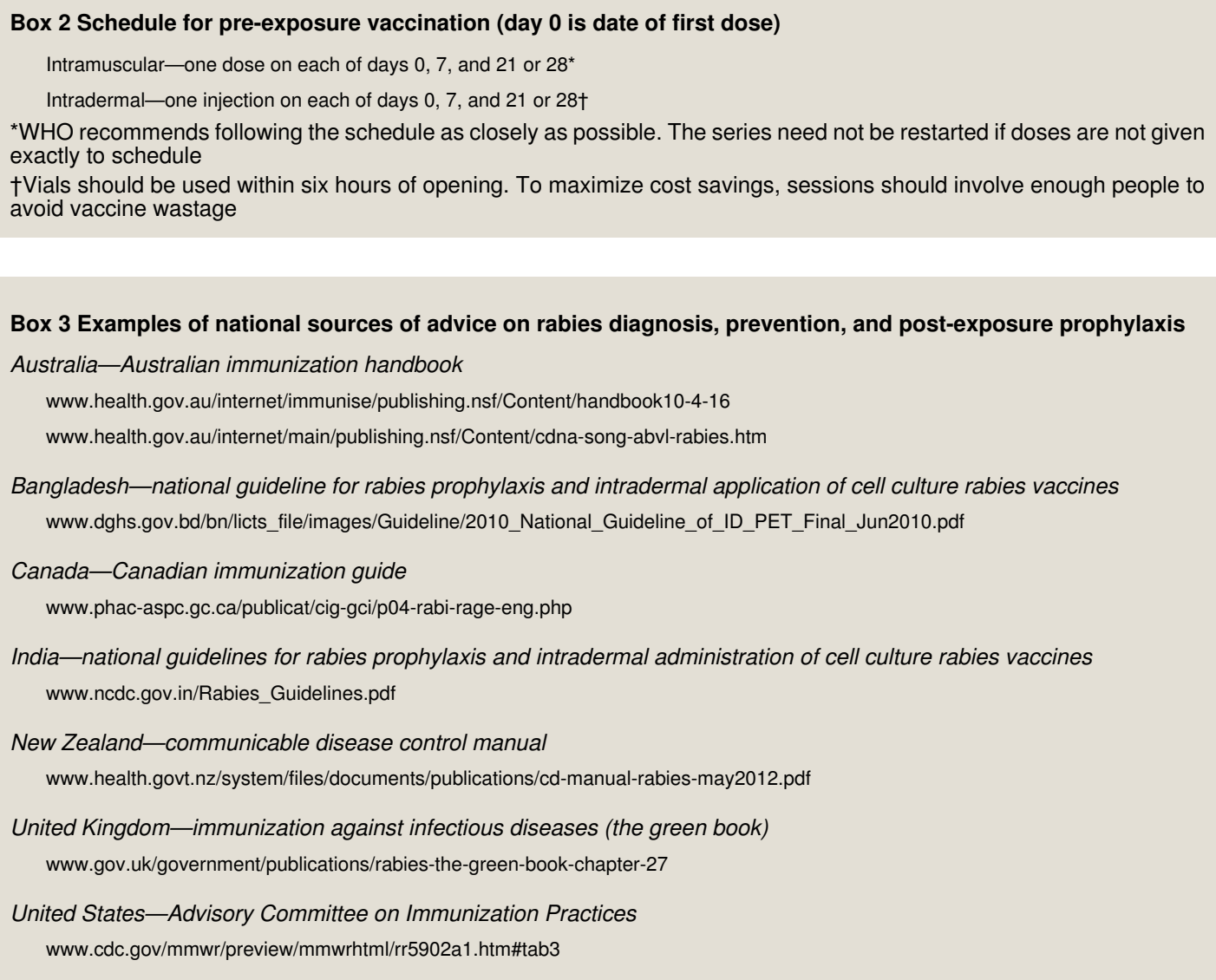

dose is $20 \mathrm{IU} / \mathrm{kg}$. As far as possible, all of the dose should be administered locally around the wound.

\section{What are the symptoms of rabies?}

Clinicians suspecting rabies should immediately contact public health authorities and the relevant reference laboratory for advice.

The incubation period after a bite may be as short as a few days or as long as years, and depends on the animal, viral inoculums, and location of the bite. ${ }^{34}$ However, most cases present within the first two months after inoculation. Prodromal symptoms are often non-specific, resembling systemic viral infections, although there may be initial neuropathic pain at the site of the bite or weakness of the affected limb. Signs suggestive of rabies include intense pruritus, beginning at the site of the bite and progressing to involve the limb or side of the face, and myoedema, a mounding of the muscle elicited by being struck with a reflex hammer and that resolves within seconds. ${ }^{35} 36$

Prodromal symptoms are quickly followed by the acute neurological phase, when the virus manifests itself in the central nervous system. This phase is referred to as paralytic or furious rabies (box 4), and progression towards coma and death occurs within one to two weeks from the onset of neurological dysfunction. ${ }^{37}$ Furious rabies, which affects two thirds of patients, is characterized by persistent fever, agitation, confusion, and seizures, and it is distinguished from other forms of encephalitis by the presence of hydrophobia, aerophobia, hypersalivation, and dysphagia. ${ }^{37}$ Patients with paralytic rabies do not present with the cardinal symptoms seen in those with the furious form and may have early features such as piloerection and fasciculations. They may also present with an ascending paralysis or symmetric quadriparesis; however, they can be distinguished from Guillain-Barré syndrome by the presence of persistent fever, an intact sensation except at the bite site, myoedema, and bladder dysfunction. Other manifestations are also being increasingly recognized, especially among patients with bat related rabies, including tremor, myoclonus, and cranial nerve, motor, or sensory deficits, which may contribute to under-reporting. ${ }^{35} 38$

The diagnosis is made clinically and confirmed by nuchal skin biopsy, with viral antigens or RNA detected at the base of hair follicles containing peripheral nerves, saliva, or brain tissue, the last submitted post mortem. ${ }^{2}$ Neutralizing antibodies in the serum may be detected seven or eight days after the onset of clinical symptoms, although they may not develop at all, ${ }^{38}$ and are occasionally found in cerebrospinal fluid.

\section{Are any treatments available for rabies?}

Death is almost always inevitable in unimmunized patients; only supportive measures are recommended after the onset of neurological signs and symptoms. The "Milwaukee protocol" is a controversial intensive care strategy that was developed for a patient who survived a bat bite despite not having received post-exposure prophylaxis and presenting with encephalitis. ${ }^{39}$ Numerous patients have subsequently failed this experimental protocol, which involves antiviral therapy and induction of a therapeutic coma to maintain a burst suppression pattern on the electroencephalogram. ${ }^{40}$ Suggested favorable factors for initiation of aggressive treatment include young age and normal immune status, receipt of rabies vaccine before the onset of neurological illness, mild neurological disease at the time of presentation, presence of anti-rabies virus neutralizing antibodies in the serum and cerebrospinal fluid early in the course of illness, and infection by a bat rabies variant, which may be associated with paralytic rabies and thus detectable serum antibodies. ${ }^{38}$ 


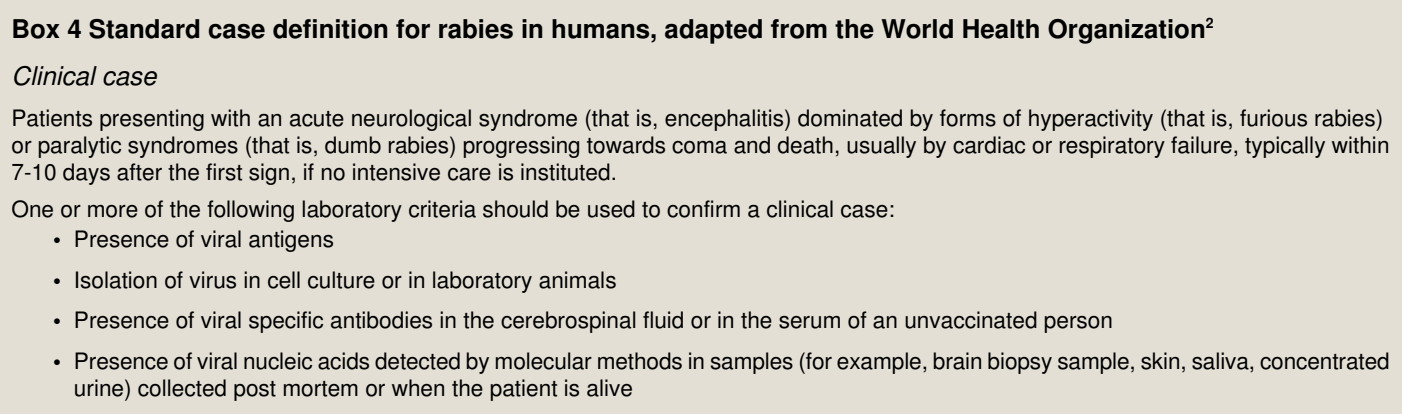

For patients admitted to hospital with rabies encephalitis, palliative measures include sedation and physical and emotional support, as such patients tend to be severely agitated and anxious. A private room is recommended to provide these measures; however, additional barrier precautions are not required as the virus is transmitted through a break in skin and not through inhaled droplets or contact with blood or faeces. Hospital contacts of patients with rabies do not require post-exposure prophylaxis unless they are bitten or their mucous membranes or any open wounds come into contact with the saliva, cerebrospinal fluid, or brain tissue of affected patients. ${ }^{2}$

\section{Are any new treatment or prevention strategies on the horizon?}

Antiviral drugs with in vitro activity against rabies virus include ribavirin and interferon-alpha, but they have shown limited activity in the setting of infection; other antiviral treatments for rabies are not on the near horizon. To tackle the global shortage of rabies immunoglobulin, clinical trials are under way to evaluate a human monoclonal antibody and a cocktail of humanized mouse monoclonal antibody. ${ }^{2} \mathrm{New}$ vaccines, including a less expensive vaccine for post-exposure prophylaxis, new delivery systems for intradermal vaccines, and dose sparing adjuvants for humans are early in development. ${ }^{122}$

Priorities for research include multi-level and multi-sectoral population and public health research into interventions aimed at policy makers, health systems, animal health, and the public for achieving elimination of rabies in dogs and optimizing delivery of pre-exposure prophylaxis and post-exposure prophylaxis to humans. ${ }^{11}$

\section{What is the advice for travelers to rabies endemic countries?}

Travelers to areas where rabies is enzootic should be aware of the risks and whether appropriate post-exposure prophylaxis will be available at their destination. ${ }^{41}$ In a review of recent cases, immigrants who had traveled home to visit family and friends, including for short trips, seemed to be at higher risk, with delays in post-exposure prophylaxis. ${ }^{17}$ Pre-exposure vaccination should be considered by travelers to endemic areas who are likely to come in contact with animals, or short term travelers making repeated visits. Short term travelers to rabies endemic countries with ready access to medical care while traveling may choose not to have pre-exposure vaccination as the risk is lower, immunization is expensive, and pre-exposure vaccination is often not publicly funded or covered by health insurance. Such people need to be fully informed about what to do in case of potential exposure to rabies during their trip.
When travelers to endemic countries seek advice too late to complete a course of post-exposure prophylaxis, they need to be aware that full post-exposure vaccination must be sought in case of a high likelihood of exposure to rabies. Options to be considered include the intradermal single visit regimen, if available; traveling with an incomplete series to be completed on return; or completing the series on arrival at the destination.

We thank PHO Library Services and Allison Crehore for help with the literature review, Winsley Rose for advice on treatment of patients, Kwame McKenzie for assistance with reviewing drafts, and the reviewers for their expert comments. This article is dedicated to the memory of Declan McKeever.

Contributors: NSC conducted the literature search and wrote the article. NT contributed to the section on rabies treatment and clinical parts of the article. Both authors contributed to the final draft for submission and are the guarantors.

Competing interests: We have read and understood the BMJ policy on declaration of interests and declare the following interests: none.

Provenance and peer review: Commissioned; externally peer reviewed.

Wunner WH, Briggs DJ. Rabies in the 21 century. PLoS Negl Trop Dis 2010;4:e591.

2 World Health Organization. WHO expert consultation on rabies: second report. WHO Press, 2013.

3 Nel LH. Discrepancies in data reporting for rabies, Africa. Emerg Infect Dis 2013;19:529-33.

4 Fitzpatrick MC, Hampson K, Cleaveland S, Mzimbiri I, Lankester F, Lembo T, et al. Cost-effectiveness of canine vaccination to prevent human rabies in rural Tanzania. Ann Intern Med 2014;160:91-100.

5 Shim E, Hampson K, Cleaveland S, Galvani AP. Evaluating the cost-effectiveness of rabies post-exposure prophylaxis: a case study in Tanzania. Vaccine 2009;27:7167-72.

6 Rumana R, Sayeed AA, Basher A, Islam Z, Rahman MR, Faiz MA.. Perceptions and treatment seeking behavior for dog bites in rural Bangladesh. Southeast Asian J Trop Med Public Health 2013;44:244-8.

7 De Serres G, Skowronski DM, Mimault P, Ouakki M, Maranda-Aubut R, Duval B. Bats in the bedroom, bats in the belfry: reanalysis of the rationale for rabies postexposure prophylaxis. Clin Infect Dis 2009;48:1493-9.

8 Tsiodras S, Dougas G, Baka A, Billinis C, Doudounakis S, Balaska A, et al. Re-emergence of animal rabies in northern Greece and subsequent human exposure, October 2012-March 2013. Euro Surveill 2013;18:20474

9 Murray CJ, Vos T, Lozano R, Naghavi M, Flaxman AD, Michaud C, et al. Disability-adjusted life years (DALYs) for 291 diseases and injuries in 21 regions, 1990-2010: a systematic analysis for the Global Burden of Disease Study 2010. Lancet 2012;380:2197-223.

10 Lembo T, Hampson K, Kaare MT, Ernest E, Knobel D, Kazwala RR, et al. The feasibility of canine rabies elimination in Africa: dispelling doubts with data. PLoS Negl Trop Dis 2010;4:e626.

11 Zinsstag J. Towards a science of rabies elimination. Infect Dis Poverty 2013;2:22.

12 Public Health England. Rabies. 2013. www.hpa.org.uk/Topics/InfectiousDiseases/ InfectionsAZ/Rabies/Generallnformation/.

13 Vigilato MA, Cosivi O, Knöbl T, Clavijo A, Silva HM. Rabies update for Latin America and the Caribbean. Emerg Infect Dis 2013;19:678-9.

14 Public Health Laboratory Service. Fatal infection with European bat lyssavirus rabies-related virus in Scotland. Commun Dis Rep CDR Wkly 2002;12(48). www.hpa.org. uk/cdr/archives/2002/cdr4802.pdf.

15 Johnstone J, Saxinger L, McDermid R, Bagshaw S, Resch L, Lee B, et al. Human rabies-Alberta, Canada, 2007. MMWR Morb Mortal Wkly Rep 2008:57:197-200.

16 Helmick CG, Tauxe RV, Vernon AA. Is there a risk to contacts of patients with rabies? Rev Infect Dis 1987;9:511-8.

17 Carrara P, Parola P, Brouqui $P$, Gautret P. Imported human rabies cases worldwide, 1990-2012. PLoS Negl Trop Dis 2013;7:e2209.

18 Bourhy H, Dautry-Varsat A, Hotez PJ, Salomon J. Rabies, still neglected after 125 years of vaccination. PLoS Neglect Trop Dis 2010;4:e839.

19 Centers for Disease Control and Prevention. Rabies in a laboratory worker-New York. MMWR Morb Mortal Wkly Rep 1977;26:183-4.

20 Winkler WG, Fashinell TR, Leffingwell L, Howard P, Conomy P. Airborne rabies transmission in a laboratory worker. JAMA 1973;226:1219-21. 


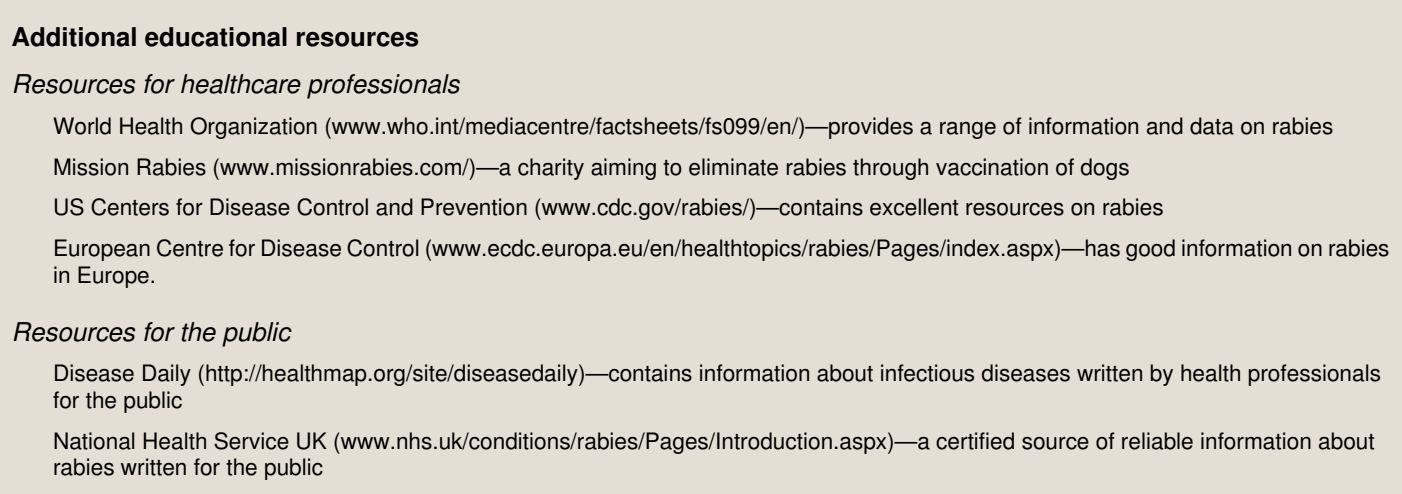

21 Both L, Banyard AC, van Dolleweerd C, Horton DL, Ma JK, Fooks AR. Passive immunity in the prevention of rabies. Lancet Infect Dis 2012;12:397-407.

22 Madhusudana SN, Mani RS. Intradermal vaccination for rabies prophylaxis: conceptualization, evolution, present status and future. Expert Rev Vaccines 2014:13:641-55.

23 Kaul DR. Donor-derived infections with central nervous system pathogens after solid organ transplantation. JAMA 2013;310:378-9.

24 Bronnert J, Wilde H, Tepsumethanon V, Lumlertdacha B, Hemachudha T. Organ transplantations and rabies transmission. J Travel Med 2007;14:177-80.

25 Vora NM, Basavaraju SV, Feldman KA, Paddock CD, Orciari L, Gitterman S, et al. Raccoon rabies virus variant transmission through solid organ transplantation. JAMA 2013:310:398-407.

26 Wilde H. Failures of post-exposure rabies prophylaxis. Vaccine 2007;25:7605-9.

27 Wilde H, Sirikawin S, Sabcharoen A, Kingnate D, Tantawichien T, Harischandra PA, et al. Failure of postexposure treatment of rabies in children. Clin Infect Dis 1996;22:228-32.

28 Maier T, Schwarting A, Mauer D, Ross RS, Martens A, Kliem V, et al. Management and outcomes after multiple corneal and solid organ transplantations from a donor infected with rabies virus. Clin Infect Dis 2010;50:1112-9.

29 Morimoto K, Patel M, Corisdeo S, Hooper DC, Fu ZF, Rupprecht CE, et al. Characterization of a unique variant of bat rabies virus responsible for newly emerging human cases in North America. Proc Natl Acad Sci U S A 1996;93:5653-8.

30 Rupprecht CE, Briggs D, Brown CM, Franka R, Katz SL, Kerr HD, et al. Use of a reduced (4-dose) vaccine schedule for postexposure prophylaxis to prevent human rabies: recommendations of the advisory committee on immunization practices. MMWR Recomm Rep 2010;59(RR-2):1-9.

31 Bernard KW, Smith PW, Kader FJ, Moran MJ. Neuroparalytic illness and human diploid cell rabies vaccine. JAMA 1982;248:3136-8.
32 Knobel DL, Cleaveland S, Coleman PG, Fèvre EM, Meltzer MI, Miranda ME, et al. Re-evaluating the burden of rabies in Africa and Asia. Bull World Health Organ 2005;83:360-8.

33 Sudarshan MK, Mahendra BJ, Madhusudana SN, Ashwoath Narayana DH, Rahman A, Rao NS, et al. An epidemiological study of animal bites in India: results of a WHO sponsored national multi-centric rabies survey. J Commun Dis 2006;38:32-9.

34 Smith JS, Fishbein DB, Rupprecht CE, Clark K. Unexplained rabies in three immigrants in the United States. A virologic investigation. N Engl J Med 1991;324:205-11.

35 Hemachudha T, Laothamatas J, Rupprecht CE. Human rabies: a disease of complex neuropathogenetic mechanisms and diagnostic challenges. Lancet Neurol 2002;1:101-9.

36 Hemachudha T, Phanthumchinda K, Phanuphak P, Manutsathit S. Myoedema as a clinical sign in paralytic rabies. Lancet 1987;1:1210.

37 Noah DL, Drenzek CL, Smith JS, Krebs JW, Orciari L, Shaddock J, et al. Epidemiology of human rabies in the United States, 1980 to 1996. Ann Intern Med 1998;128:922-30.

38 Udow SJ, Marrie RA, Jackson AC. Clinical features of dog- and bat-acquired rabies in humans. Clin Infect Dis 2013;57:689-96.

39 Willoughby RE Jr, Tieves KS, Hoffman GM, Ghanayem NS, Amlie-Lefond CM, Schwabe $\mathrm{MJ}$, et al. Survival after treatment of rabies with induction of coma. $N$ Engl $J$ Med 2005;352:2508-14.

40 Jackson AC. Current and future approaches to the therapy of human rabies. Antiviral Res 2013;99:61-7.

41 Jentes ES, Blanton JD, Johnson KJ, Petersen BW, Lamias MJ, Robertson K et al. The global availability of rabies immune globulin and rabies vaccine in clinics providing indirect care to travelers. J Travel Med 2014;21:62-6.

Cite this as: $B M J$ 2015;350:97827

๑ BMJ Publishing Group Ltd 2015 


\section{Tables}

\section{Table 1| Recommended post-exposure regimens for rabies. Adapted from World Health Organization ${ }^{2}$}

Route

Schedule (day 0 is date of first dose)

For non-immunized or incompletely immunized people

Intramuscular:

5 dose Essen regimen

One intramuscular dose on each of days $0,3,7,14$, and 28 plus rabies immunoglobulin for category III exposures; in immunocompetent people, a reduced course with four vaccine doses on days $0,3,7$, and 14 may be considered, provided they receive wound care plus rabies immunoglobulin for both categories II and III exposures and a WHO prequalified rabies vaccine is used

4 dose Zagreb regimen

Two doses of vaccine on day 0 , one dose on days 7 and 21

Intradermal*:

Thai Red Cross regimen

Two intradermal doses of $0.1 \mathrm{~mL}$ vaccine at two different sites on days $0,3,7$, and 28

For people with documented previous complete pre-exposure or post-exposure prophylaxis with rabies CCEEVs†

Intramuscular

One intramuscular dose at one site on both days 0 and 3 (no rabies immunoglobulin required) $\ddagger$

Intradermal

"One visit four-site" intradermal regimen. Four injections of $0.1 \mathrm{~mL}$ equally distributed over left

and right deltoids, thigh, or suprascapular areas at one visit

CCEEVs=cell culture and embryonated egg-based rabies vaccines.

*WHO states that this regimen can be used for people with category II or III exposure in countries in which the intradermal route has been endorsed by the national health authorities.

†People with category III exposures who have received complete pre-exposure or post-exposure prophylaxis with a vaccine of unproved potency should be managed as if unvaccinated and receive a full post-exposure vaccination course, including rabies immunoglobulin.

$\ddagger$ This regimen can also be given to people vaccinated against rabies who have detectable rabies virus neutralizing antibody. 
Table 2| Decision aid for post-exposure prophylaxis according to type of exposure. Adapted from World Health Organization ${ }^{2}$

\begin{tabular}{|c|c|c|}
\hline Category of exposure & $\begin{array}{c}\text { Type of exposure to domestic or wild }{ }^{\star} \text { animal suspected } \\
\text { or confirmed to be rabid, or animal unavailable for } \\
\text { testing }\end{array}$ & Recommended post-exposure prophylaxis \\
\hline I & $\begin{array}{l}\text { Touching or feeding animals; licks on intact skin; contact } \\
\text { of intact skin with secretions or excretions of rabid animal } \\
\text { or human case }\end{array}$ & None, if reliable case history is available \\
\hline II & $\begin{array}{l}\text { Nibbling of uncovered skin; minor scratches or abrasions } \\
\text { without bleeding }\end{array}$ & $\begin{array}{l}\text { Administer vaccine immediately } \nmid \text { ' stop treatment if animal remains } \\
\text { healthy throughout an observation period of } 10 \text { days } \neq \text { or is proved } \\
\text { to be negative for rabies by reliable laboratory using appropriate } \\
\text { diagnostic techniques }\end{array}$ \\
\hline III & $\begin{array}{l}\text { Single or multiple transdermal bites§ or scratches, licks on } \\
\text { broken skin; contamination of mucous membrane with saliva } \\
\text { (that is, licks) exposure to bats } \uparrow\end{array}$ & $\begin{array}{l}\text { Administer rabies vaccine immediately, and rabies immunoglobulin, } \\
\text { preferably as soon as possible after initiation of post-exposure } \\
\text { prophylaxis. Rabies immunoglobulin can be injected up to seven } \\
\text { days after first vaccine dose has been administered. Stop treatment } \\
\text { if animal remains healthy throughout an observation period of } 10 \\
\text { days (does not apply to bats) or is proved to be negative for rabies } \\
\text { by a reliable laboratory using appropriate diagnostic techniques }\end{array}$ \\
\hline
\end{tabular}

*Post-exposure prophylaxis not routinely required for exposure to rodents, rabbits, or hares.

†Treatment may be delayed if an apparently healthy dog or cat is from a low risk area and placed under observation.

$\ddagger$ Applies to dogs and cats only. Except for threatened or endangered species, other domestic and wild animals suspected of being rabid should be euthanized and their tissues examined for the presence of rabies antigen by appropriate laboratory techniques.

§Bites especially on the head, neck, face, hands, and genitals are category III exposures because of the rich innervation of these areas.

IPost-exposure prophylaxis should be considered when contact between a human and a bat has occurred, unless the exposed person can rule out a bite or scratch or exposure of a mucous membrane. 


\section{Figures}

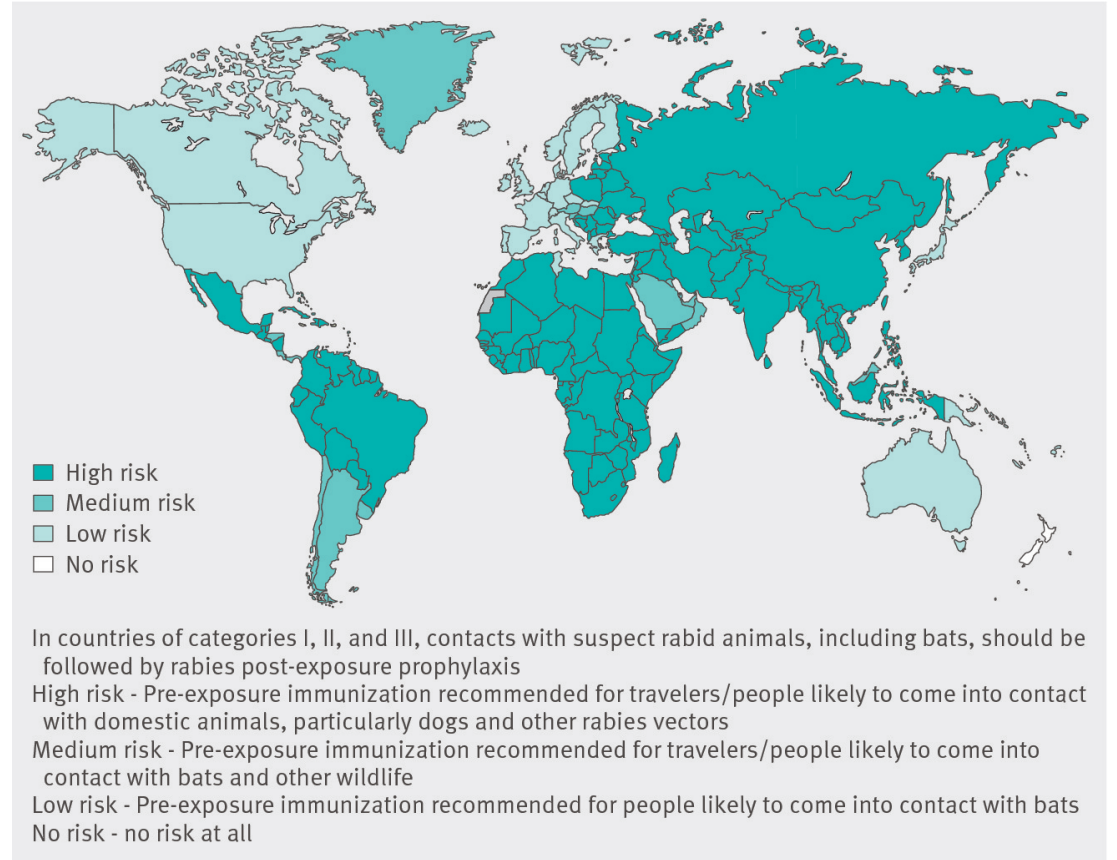

Fig 1 Map of rabies endemic areas

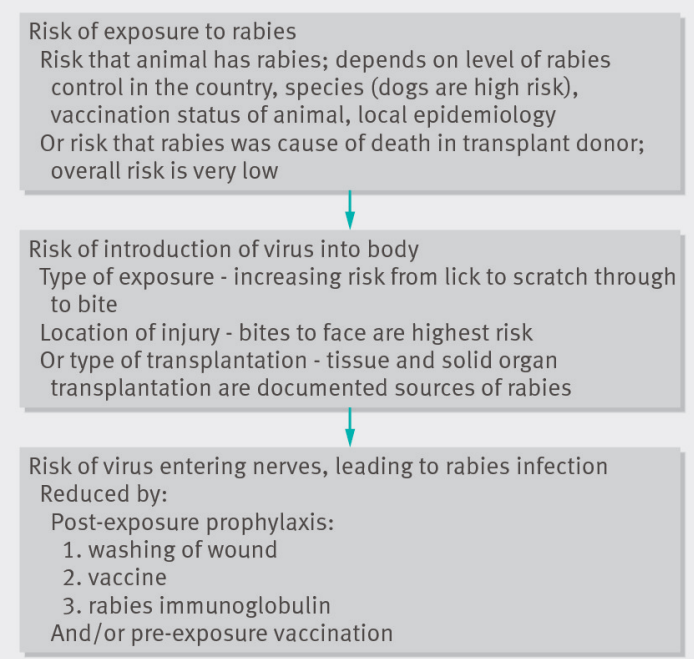

Fig 2 Components involved in risk of acquiring rabies 